\title{
Visual Cortex Inspired Pixel-Level Re-configurable Processors for Smart Image Sensors
}

\author{
Pankaj Bhowmik $^{\dagger}$, Md Jubaer Hossain Pantho ${ }^{\dagger}$, Christophe Bobda ${ }^{\ddagger}$ \\ $\dagger$ Department of Computer Science and Computer Engineering, University of Arkansas \\ ¥Department of Electrical and Computer Engineering, University of Florida \\ †pbhowmik|mpantho@uark.edu, ${ }^{\ddagger}$ cbobda@ece.ufl.edu
}

\begin{abstract}
This paper presents a reconfigurable hardware architecture of smart image sensors to speed up low-level image processing applications at the pixel level. For each pixel in the sensor plane, the design includes an activation module and a processor. The processor has a basic structure which is common to all applications and reconfigurable segments for specific applications. Visual cortex inspired computing, like, Predictive Coding in time is implemented in the activation module to remove temporal redundancy. The ASIC implementation shows the design saves up to $84.01 \%$ dynamic power and achieves $9 \mathrm{x}$ speedup at $800 \mathrm{MHz}$ by accurate prediction.
\end{abstract}

\section{INTRODUCTION}

To meet the increasing demand for high-speed real-time machine vision applications, smart image sensors (SIS) bring the computational units (XPU) close to pixel circuit to perform low-level image processing in parallel [1]. Usually, the pixel circuits are comprised of per-pixel ADC with a corresponding memory [3] [4] and the SIS combines a smart part after this memory to give pixel-level parallelism. One of the difficulties in the SIS is high power consumption since all XPUs are functional throughout the entire operation. $\mathrm{Vi}$ sual cortex inspired circuits are one of the solutions to mitigate this obstacle. In our vision system, the retina receives pixels in parallel, removes temporal redundancy, and executes low-level operations. Predictive Coding in time (PCT) defines the process of how the visual cortex removes the temporal redundancies [2]. This concept can be implemented in a circuit which can effectively remove the redundant information. On the other hand, the smart part in SIS becomes application specific after fabrication and is another limitation. This part needs to be reconfigurable to provide greater functionality with simpler hardware. The cost of supporting additional features introduces some overhead but presents a flexible design. With this flexibility, the system will be capable to run the required low-level processing at the pixel level to accelerate desired machine vision applications. In this paper, we propose an XPU for each pixel by emulating the visual cortex. Here, we implement predictive coding in time (PCT) to remove temporal redundancy

Permission to make digital or hard copies of all or part of this work for personal or classroom use is granted without fee provided that copies are not made or distributed for profit or commercial advantage and that copies bear this notice and the full citation on the first page. Copyrights for components of this work owned by others than ACM must be honored. Abstracting with credit is permitted. To copy otherwise, or republish, to post on servers or to redistribute to lists, requires prior specific permission and/or a fee. Request permissions from permissions@acm.org.

DAC '19, June 2-6, 2019, Las Vegas, NV, USA

(C) 2019 Association for Computing Machinery.

ACM ISBN 978-1-4503-6725-7/19/06 ..\$15.00

https://doi.org/10.1145/3316781.3322481

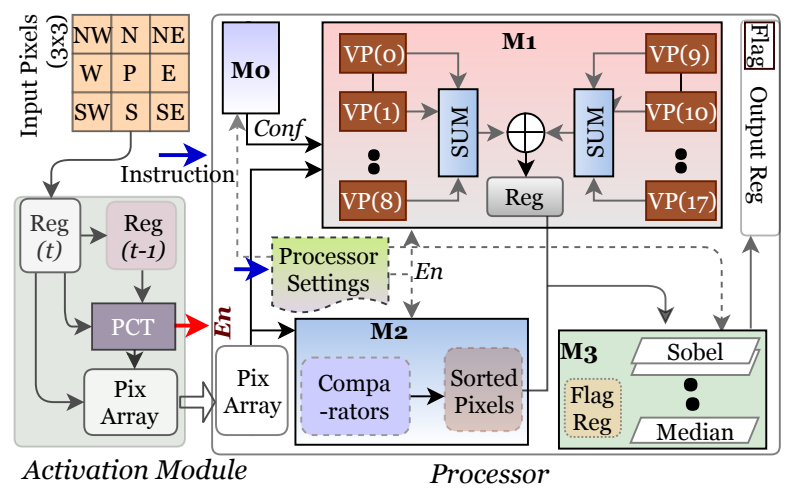

Figure 1: The functional block diagram of the proposed Reconfigurable Processor for each pixel.

and a reconfigurable processor to perform a set of low-level operations to ensure flexibility. The design achieves high acceleration and mitigates the computational burden for a sequential processor.

\section{PROPOSED ARCHITECTURE}

We start our architecture assuming that we have an image sensor with a per-pixel 8-bit resolution ADC and the intensity value is temporarily stored to the corresponding register [4]. The proposed processing units (XPU) are integrated after the register to perform pixel-level processing in parallel. We present the basic block diagram of the XPU in Figure 1. It has two separate modules and their detailed descriptions are provided below.

Activation Module (ACM): In this module, we implement the PCT to predict the pixels in the $3 \times 3$ window for the corresponding pixel $\mathrm{P}$ as shown in Figure 1 . The PCT module takes the inputs at the time $t$ and ( $t-1)$ from two register arrays. This module predicts the current pixel values with the pixels values at $(t-1)$. If there is no significant change observed between the predicted values and the original values then we consider the prediction is accurate and there is no temporal change. With an accurate prediction, PCT sends zero to the En port of the processor and one otherwise. The pixel array block sends the pixel values to the processor in parallel if the PCT produces signal one.

Processor: The proposed processor is a reconfigurable unit intended to handle low-level image processing in parallel. We propose an algorithm to support a set of low-level algorithms. The algorithm has a basic structure which is common to all applications, and a separate structure to support unique portions in each application. In Figure 1, M1 module performs convolution for the input image with two convolutional-kernels using vector processors (VP) and M0 module assigns appropriate kernel values. For the $3 \times 3$ window, 
M1 contains 18 VPs for parallel execution. To be configured for different applications, M0 generates a new set of kernels. Alternately, with the M2 module, the processor performs nonlinear filtering (min, median, max filters). It is comprised of a set of comparators to perform sorting and arranging the pixel intensities in an ascending form. After sorting, we can obtain the min, max, or mid values for further processing. M1 and M2 module jointly execute the common segment of the low-level algorithms. The remainder operation is processed by the module M3. It has separate sections for every application to execute their unique operations. Once the process in M3 finishes, it sets the tag value and resets otherwise. M3 keeps the output with the tag on the output register. In this figure, the processor setting block is responsible to decode the input instruction from the general purpose input pins and to create proper signaling for M0 M4 modules to adapt the configuration to support a set of applications. To execute a convolution-based operation, it enables M1 and M0 module. At the same time, it gives another signal to the M3 to inform which particular task needs to accomplish. On the other hand, M2 is selected to run nonlinear filtering.

Overall Functionality and Benefits: When the ACM provides zero to the $E n$ of the processor, the processor postpones the execution. In this case, the circuits physically remain in the design but they become logically disabled. They draw leakage power only and the output register holds the previous value. This procedure removes the computation on the same set of pixels and we can truncate the temporal redundancy which saves power and time. Alternately, if the $E n$ of the processor receives one, it resumes the operation and the output. In addition, changing the instruction, processor settings can be altered to run a different application. When the low-level operations are performed at the pixel level, the remaining highlevel operations of a machine vision application can be performed on a sequential processor.

\section{EXPERIMENTAL RESULTS}

We implement the full RTL-to-GDSII flow on ASIC for the XPUs at the block level using $1.1 \mathrm{~V}$ supply voltage and $800 \mathrm{MHz}$ clock frequency in $45 \mathrm{~nm}$ technology.

We implement PCT [2] and the proposed algorithm who supports twelve low-level image processing applications (listed in Table 1) in the activation module and processor, respectively. On the aforementioned platform, we firstly implement these modules separately for evaluating their individual performance and tabulate them in Table 2. Analyzing this table we can state that, PCT costs only $14.21 \%$ power and $11.72 \%$ area overhead. But when it makes the right prediction or if there is no temporal change, the processor draws leakage power only which gives up to $84.01 \%$ power saving with $9 \times$ speedup from each XPU. Hence, the integration of ACM with the processor is a viable approach to save power. However, the architecture is scalable to integrate any number of XPUs. In Figure 2 we show the impact of ACMs on multiple XPUs run in parallel. These results represent that, when these XPUs are driven without ACMs, they consume the same amount of power all the time. By adding ACMs, we make power consumption as a function of prediction or temporal change. The bar-charts give the estimations of power saving for different prediction rates from multiple XPU groups. In the case, where every pixel in the XPU group experience temporal change, a design without ACM shows power efficient.
Table 1: List of Image processing applications run on XPU

\begin{tabular}{l|l} 
Convolution-Based & Sorting-Based \\
\hline Edge detection (Sobel, Laplacian, FeiChen, & Min filter, Max filter, \\
Prewitt), Neighborhood Averaging, & Median filter, \\
Gaussian Smoothing, Image sharpening & Local Thresholding
\end{tabular}

Table 2: Parameter Extraction of the Modules

\begin{tabular}{c|c|c|c|c|c|c} 
& $\begin{array}{c}\text { Area } \\
(\mathrm{um} 2)\end{array}$ & $\begin{array}{c}\text { Power } \\
(\mathrm{mW})\end{array}$ & $\begin{array}{c}\text { Leakage } \\
(\mathrm{uW})\end{array}$ & $\begin{array}{c}\text { Delay } \\
(\mathrm{ns})\end{array}$ & $\begin{array}{c}\text { Density } \\
(\%)\end{array}$ & $\begin{array}{c}\text { Wire le- } \\
\text { ngth(mm) }\end{array}$ \\
\hline ACM & 589.7 & 0.694 & 12.5 & 1.25 & 70.16 & 1.55 \\
\hline Proc & 4437.95 & 4.189 & 86.7 & 10 & 60.94 & 24.68 \\
\hline XPU & 5027.7 & 4.883 & 99.2 & 11.25 & 70.01 & 24.84 \\
\hline
\end{tabular}

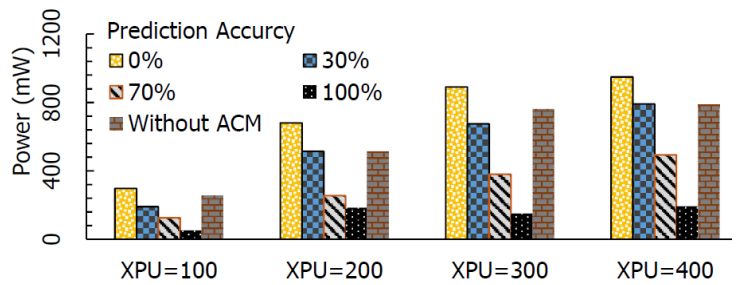

Figure 2: Dynamic Power Reduction on the basis of prediction accuracy from different number of XPUs.

Table 3: Percentage (\%) increase in Area and Power overhead in our processor w.r.t. single functioned processor.

\begin{tabular}{|c|c|c|c|c|c|}
\hline Application & Area & Power & Application & Area & Power \\
\hline Sobel & 34.65 & 18.1 & Smoothing & 68.18 & 53.01 \\
\hline Laplacian & 42.20 & 16.37 & Median Filter & 40.65 & 19.54 \\
\hline Prewitt & 35.24 & 20.11 & Max Filter & 40.72 & 19.57 \\
\hline
\end{tabular}

But a natural scene contains a huge amount of redundant information and this figure states the reduction of power consumption through prediction. Adding to it, since the algorithm is reconfigurable to twelve applications, it provides some additional area and power overhead. To evaluate our design we implement these single functioned processors and compute their individual area and power consumption. The percentage increase in area and power overheads in our design compared to those single functioned processors are tabulated in table 3. Analyzing these results, it is obvious that, these overheads stay in the acceptable range.

\section{CONCLUSION}

The proposed XPU in the SIS leverages the concept of the visual cortex and allows a set of low-level applications to be executed on the reconfigurable processors to accelerate machine vision applications. Using PCT the architecture saves $84.01 \%$ power from each smart part of the SIS with $9 \times$ speedup when there is no temporal change or making the right prediction.

\section{ACKNOWLEDGEMENT}

This work was supported by the NSF under Grant-1618606.

\section{REFERENCES}

[1] Jérôme Dubois et al. 2008. A 10000 fps CMOS sensor with massively parallel image processing. IEEE fournal of Solid-State Circuits 43, 3 (2008), 706-717.

[2] Yanping Huang and Rajesh PN Rao. 2011. Predictive coding. Wiley Interdisciplinary Reviews: Cognitive Science 2, 5 (2011), 580-593.

[3] Stuart Kleinfelder et al. 2001. A 10000 frames/s CMOS digital pixel sensor. IEEE fournal of Solid-State Circuits 36, 12 (2001), 2049-2059.

[4] Masaki Sakakibara et al. [n. d.]. A back-illuminated global-shutter CMOS image sensor with pixel-parallel 14b subthreshold ADC. 